\title{
A HAEMORRHEOLOGICAL CHARACTERISATION OF THE NEWBORN CIRCULATION AT BIRTH
}

\author{
R.J. MacKinnon ${ }^{1}$, J. Wai ${ }^{2}$, R. Gottstein ${ }^{1}$, X.-F. Yuan ${ }^{2}$ \\ ${ }^{1}$ Royal Manchester Children's Hospital, ${ }^{2}$ Manchester Interdisciplinary Biocentre; School of Chemical \\ Engineering and Analytical Science, Manchester, UK
}

Background \& aims: To quantitatively characterise the rheological properties of newborn bloods using a Vilastic-3 rheometer.

Methods: Umbilical cord blood from 20 full term infants was characterised by Vilastic-3 rheometer and directly imaged by Linkam Imaging Station under capillary flow.

Results: Viscosity and elasticity: Viscosity remained constant over a range of shear rates. Elasticity decreased with increasing shear rates. Low shear rate $(<2 s-1)$ elasticity was linear, RBCs existed in rouleaux and large aggregates, with minimal deformability. Intermediate shear rate (2s-1 to $100 \mathrm{~s}-1)$ elasticity decreased, size of aggregates started to diminish and deformability of RBCs began to rise. High shear rate $(>100 \mathrm{~s}-1)$, elasticity dropped further, aggregates disappeared and deformability of red cells was dominant.

Effect of haematocrit: Relatively uniform increase in visco-elasticity properties with increase haematocrit. Only divergent sample was the only infant admitted to intensive care with breathing difficulty.G' and G": newborn blood cannot be fitted by a single-mode Maxwell model. The slopes of G" show the signature of non-Newtoian fluids.
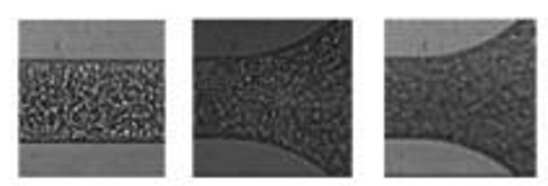

[Rouleaux and increasing shear rate (right to left)]

Conclusions: This study quantitatively charcterised the flow behaviour of newborn blood and their rheological properties, including viscosity, elasticity, storage and loss moduli, as well as the structural evolution of rouleaux, aggregation \& deformation of red blood cells. These rheological properties have also been identified as potential marker of pathology. 